\title{
Wavelet Characterization of Ozone Layer Depletion for Atmospheric Region of Pakistan
}

\author{
M. Ayub Khan Yousuf Zai*, M. Rashid Kamal Ansari, Jawaid Iqbal, Arif Hussain, and Ahmed Hasan \\ Institute of Space and Planetary Astrophysics and Department of Applied Physics, University of Karachi, Karachi, \\ 75270, Pakistan Institute of Mathematical Sciences, Federal University of Arts, Science and Technology, Karachi, \\ Pakistan
}

\begin{abstract}
Ozone is of considerable importance to mankind because of its role as a shield for the biosphere against the Ultraviolet (UV) radiation from the outer space, in particular from the sun. In the recent years the advancement in the science and technology has increased the understanding of various phenomena of the environment and consequently the predictability of many naturally occurring events. It has been Due to a serious reduction of O3 content in the atmosphere life-forms on the earth are exposed to a new hazard in the form of an increase of harmful solar UV radiation intensity at the sea level. Obviously, this situation calls for an assessment, monitoring and prevention of the incidence of decrease in the $\mathrm{O} 3$ concentration. The fluctuating dynamics of stratospheric ozone layer depletion (OLD) for Pakistan atmospheric region has been introduced by mentioning the production and annihilation of ozone. The recent development in the theory and application of wavelets are yielding powerful multi resolution techniques for the analysis of time series and image data. Wavelets are the central idea of a broad framework for displaying and analyzing data. Wavelets possess a simultaneous time-frequency localization that makes them useful in presenting complicated signals. In this communication behaviour of ozone is explained on the basis of ozone profile, effects of periodic, a-periodic change along with effects of monthly and seasonal variations using wavelet analysis.
\end{abstract}

\section{Introduction}

Ozone in the stratosphere acts to protect us from harmful ultraviolet radiation from the sun that it absorbs. It has been inspected that a small decrease in the ozone concentration can lead to a large increase in the amount of ultraviolet (UV) reaching Arabian sea specially around wavelength range of 290$320 \mathrm{~nm}$. Excessive UV exposure can cause skin cancers and in large amounts can also harmful to plant growth. The highest risks are in spring at extreme southerly latitudes or to a lesser extent at extreme northerly latitudes, corresponding to the formation of the ozone holes over Antarctic and Arctic polar regions.The ozone layer is also a separate issue from climate change and greenhouse warming. The $\mathrm{CFC}$ that cause ozone layer depletion are greenhouse gases, but destruction of the ozone layer reduces the greenhouse effect slightly as the ozone also acts as greenhouse gas.

${ }^{*}$ Corresponding author

E-mail: ayubzai@yahoo.com

(C) 2015 International Association for Sharing Knowledge and Sustainability

DOI: $10.5383 /$ swes.7.01.007
Conversely, if the ozone destruction were to become serious enough to reduce primary productivity of phytoplankton and forest ecosystems, as a result of UV damage, this would affect climate change. As with many environmental systems, all are interconnected. The ozone (O3) forms from oxygen by reaction under the influence of UV radiation. Shorter wavelength radiation, of wavelength $175 \mathrm{~nm}$ or less, has sufficient energy to dissociate oxygen into two separate free oxygen atoms. This occurs at heights of about 50-60- $\mathrm{km}$ and results in very little radiation this wavelength penetrating further. The free $\mathrm{O}$ atom can then combine with $\mathrm{O} 2$ molecule to form Ozone. The ozone forms in this above tropical and equatorial regions where solar radiation is highest, and spreads around the globe to form this layer at between 20 and $26 \mathrm{~km}$ in height. 
The ozone layer if expressed at standard temperature and pressure would be $3 \mathrm{~mm}$ thick, yet it absorbs strongly enough in the Uv region a to act as an almost complete shield against radiation of wavelength less than around $295 \mathrm{~nm}$.

$$
\mathrm{O} 2 \rightarrow 2 \mathrm{O}
$$

Oxygen dissociates in presence of UV radiation

$$
\mathrm{O} 2+\mathrm{O} \rightarrow \mathrm{O} 3
$$

Ozone formation

However, in the presence of certain pollutants, chiefly chlorofluorocarbons (CFC)s, ozone can be destroyed. Free chlorine $(\mathrm{Cl})$ radicals acts to strip $\mathrm{O} 3$ of an oxygen atom, forming $\mathrm{O} 2$ and $\mathrm{ClO}$ that then loses the $\mathrm{O}$ to a free oxygen atom to form $\mathrm{O} 2$ and $\mathrm{Cl}$ again [3]

$$
\begin{array}{r}
\mathrm{O} 3+\mathrm{Cl} \rightarrow \mathrm{O} 2+\mathrm{ClO} \text { Ozone destruction by } \mathrm{Cl} \text { radical } \\
\mathrm{ClO}+\mathrm{O} \rightarrow \mathrm{Cl}+\mathrm{O} 2 \quad \mathrm{Cl} \text { radical free to act again }
\end{array}
$$

The net effect is to transform ozone into $\mathrm{O} 2$ while leaving the $\mathrm{Cl}$ radical free to promote another reaction thus one molecule of $\mathrm{CFC}$ can destroy a large number of ozone molecules.

Nitric Oxide and hydroxyl $(\mathrm{OH})$ radicals can act the same way. The resulting reduction in the ozone concentration depends upon a balance between the processes of formation and annihilation. The series of reactions as shown above that destroy ozone take place mostly readily at low temperatures and so occur mainly over the poles during the spring time. It has been investigated that in the region of stratosphere there is little water vapors and so no cloud. During the Antarctic winter temperatures plummet and the temperature of stratosphere drops below around $-800 \mathrm{C}$, polar stratospheric clouds can form. These clouds allow the formation of highly reactive free $\mathrm{Cl}$ radicals. In the early spring as the first rays of sun strike the clouds the reactions that destroy ozone commence. This results in the so - called ozone hole over the Antarctic that can be measured by means of remote sounding equipment where the atmospheric absorption are observed. The Antarctic ozone hole has occurred every winter for the past two decades with increasing severity. During the summer the ozone is replenished from the tropics. It has been observed that ozone loss is less severe at Arctic because stratospheric temperatures are not usually low enough to allow clouds to form, albeit in recent years an Arctic ozone hole has also formed. Reaching a record low in the year 2000 [4].

While production of the most harmful CFCs is not subject to strict international controls stemming from the Montreal Protocol, they are very long-lived once they reach the stratosphere and still being emitted from many sources. The ozone hole appeared to have deteriorated more rapidly than hoped or expected. It is thought this may be due to a feedback with climate change. Increased $\mathrm{CO} 2$ levels in the troposphere hold the Earth's heat at the surface, resulting in lowering the temperatures in the stratosphere. These lowering temperatures promote PSCs that allow OLD more rapidly. Hence the ozone holes have increased more rapidly in both size and level of depletion. Obviously, this situation calls for an assessment, monitoring and prevention of the incidence of decrease in the $\mathrm{O} 3$ concentration. In this communication we have studied to quantify the wavelet characteristic for ozone layer depletion. In order to strengthen the concepts of wavelet analysis we have introduced some ideas of Fourier Analysis, Fourier Series, Fourier Transform and fast Fourier transforms (fft) Using the
Fourier analysis, seasonal trends in atmospheric, meteorological and environmental data sets can be removed.

\section{Fourier Analysis, Fourier series and Fourier Transform}

We have introduced the basic idea of the signals then sampling the characteristics for OLD. For this purpose we have used Fourier analysis (also known as spectral analysis, frequency analysis, or harmonic analysis) that uses stationary or detrended data sets with inherent periodicities. The time series can be represented by a superposition of sines and cosines of various amplitudes and frequencies as shown in the table 1 . With this finite data set of ozone depths (1960-2002) the range of frequencies that can be discerned is limited on the low end to $f_{0}=1 / \mathrm{N}$ (the fundamental frequency) by finite length of the record and on the high end to $f_{\mathrm{c}}=\mathrm{I} / 2$ (the Nyquist frequency) by the sample rate The $f_{0}$ comes from at least one full oscillation. The Nyquist frequency from at least two data points per period to define oscillation. The reciprocal of these frequencies are the periods of the corresponding oscillations. Therefore, it can be seen from the figure.1.It depicts enhancement and depletion in the ozone layer at height ranging from $25-55 \mathrm{~km}$. Above sea level with time The data has been supplied from the year 1960 to 2002

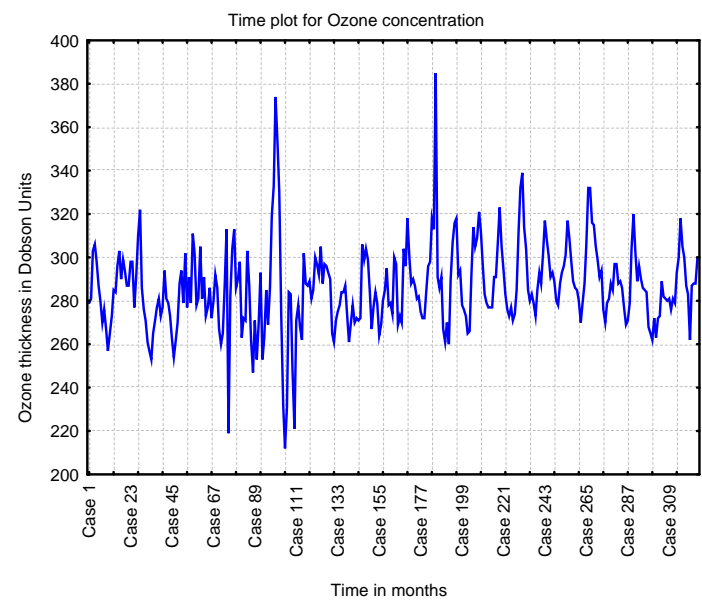

Figure 1. Temporal Fluctuation of Ozone Concentration at Pakistan's Stratosphere

A temporal variation for ozone depths is depicted in fig.1. Thus with a finite data set as mentioned in this paper we can do the best is approximate $\mathrm{Xn}$ by

$$
\mathrm{Xn}=\frac{a_{0}}{2}+\sum_{m=1}^{N / 2} a_{m} \operatorname{Cos} \frac{2 \pi m n}{N}+\mathrm{b}_{\mathrm{m}} \operatorname{Sin} \frac{2 \pi m n}{N}
$$

Where $\mathrm{N} / 2$ different frequencies or harmonics $(\mathrm{f}=\mathrm{mf} 0)$ have amplitudes

$$
a_{m}=\frac{2}{N} \sum_{n=1}^{N} X_{n} \operatorname{Cos} \frac{2 \pi m n}{N}
$$




$$
b_{m}=\frac{2}{N} \sum_{n=1}^{N} X_{n} \operatorname{Sin} \frac{2 \pi m n}{N}
$$

These equations constitute the discrete Fourier transform (DFT). The coefficients values as given in the table. with periodogram and density as well as the Hamming values they contain the data points but are in frequency domain rather than the time domain.

\section{Table.1}

\begin{tabular}{|c|c|c|c|c|c|c|}
\hline & Period & Cosine & Sine & Periodog & Density & Hamming \\
\hline $\mathbf{0}$ & & 0.00000 & 0.00000 & 0.00 & 946.24 & 0.035714 \\
\hline 1 & 324.0000 & $\overline{0} .78174$ & -3.13506 & 1691.23 & 1268.12 & 0.241071 \\
\hline 2 & 162.0000 & $\overline{1} .40758$ & 3.05367 & 1831.60 & 1355.94 & 0.446429 \\
\hline 3 & 108.0000 & 0.02650 & 1.38857 & 312.47 & 1043.71 & 0.241071 \\
\hline 4 & 81.0000 & 1.14279 & 2.87039 & 1546.31 & 1060.83 & 0.035714 \\
\hline 5 & 64.8000 & 2.13755 & 0.72683 & 825.78 & 962.25 & \\
\hline 6 & 54.0000 & 1.14936 & 1.99612 & 859.49 & 671.31 & \\
\hline 7 & 46.2857 & 0.65010 & -0.05822 & 69.02 & 417.10 & \\
\hline 8 & 40.5000 & 1.41631 & 0.93554 & 466.75 & 562.04 & \\
\hline 9 & 36.0000 & 2.13464 & -1.36061 & 1038.09 & 987.81 & \\
\hline 10 & 32.4000 & 0.85847 & -2.99259 & 1570.20 & 1311.15 & \\
\hline 11 & 29.4545 & 2.30947 & -0.01927 & 864.11 & 1793.67 & \\
\hline 12 & 27.0000 & $\overline{1}-54234$ & -4.57637 & 3778.16 & 2574.50 & \\
\hline 13 & 24.9231 & 2.89265 & -2.39115 & 2281.77 & 2651.09 & \\
\hline 14 & 23.1429 & 0.10720 & 3.55931 & 2054.18 & 3008.22 & \\
\hline 15 & 21.6000 & 5.51246 & -1.84816 & 5476.08 & 3753.03 & \\
\hline 16 & 20.2500 & 3.75735 & 0.86863 & 2409.29 & 3544.89 & \\
\hline 17 & 19.0588 & 3.60675 & 3.61439 & 4223.74 & 3094.94 & \\
\hline 18 & 18.0000 & - 0.95564 & 3.00691 & 1612.67 & 2232.03 & \\
\hline 19 & 17.0526 & $\overline{-}-52641$ & -2.71292 & 1237.20 & 1857.39 & \\
\hline 20 & 16.2000 & 1.91299 & 3.90875 & 3067.93 & 1917.68 & \\
\hline 21 & 15.4286 & $\overline{-}-1.32188$ & 1.65017 & 724.21 & 1312.44 & \\
\hline 22 & 14.7273 & $\overline{1}-40065$ & -1.04158 & 493.57 & 1095.43 & \\
\hline 23 & 14.0870 & 3.81408 & -0.61773 & 2418.46 & 1287.70 & \\
\hline 24 & 13.5000 & 0.42324 & 1.08949 & 221.31 & 824.98 & \\
\hline 25 & 12.9600 & 0.18765 & -1.28943 & 275.05 & 1715.93 & \\
\hline
\end{tabular}

\begin{tabular}{|c|c|c|c|c|c|}
\hline 26 & 12.4615 & 3.16214 & 0.48659 & 1658.22 & 8016.24 \\
\hline 27 & 12.0000 & 1.66582 & 13.39178 & 29502.58 & 14200.78 \\
\hline 28 & 11.5714 & 1.82871 & -3.48270 & 2506.69 & 8445.76 \\
\hline 29 & 11.1724 & 1.48800 & 0.75601 & 451.28 & 2208.53 \\
\hline 30 & 10.8000 & 1.13240 & 2.59775 & 1300.96 & 1028.75 \\
\hline 31 & 10.4516 & 2.27032 & -0.98937 & 993.58 & 885.95 \\
\hline 32 & 10.1250 & 0.86955 & -0.99721 & 283.59 & 740.41 \\
\hline 33 & 9.8182 & 2.64709 & -0.80382 & 1239.82 & 876.88 \\
\hline 34 & 9.5294 & $\overline{0} .19506$ & 2.22770 & 810.11 & 840.40 \\
\hline 35 & 9.2571 & 0.27790 & -2.02816 & 678.88 & 602.86 \\
\hline 36 & 9.0000 & 1.02068 & 0.08746 & 170.01 & 458.22 \\
\hline 37 & 8.7568 & 0.42376 & 1.77300 & 538.34 & 740.64 \\
\hline 38 & 8.5263 & 0.12191 & -3.21683 & 1678.79 & 1096.21 \\
\hline 39 & 8.3077 & 2.14112 & -0.81596 & 850.53 & 899.97 \\
\hline 40 & 8.1000 & 0.35863 & 0.94045 & 164.12 & 748.25 \\
\hline 41 & 7.9024 & 3.07491 & -0.59824 & 1589.70 & 1000.56 \\
\hline 42 & 7.7143 & 0.85920 & -1.97165 & 749.35 & 1009.41 \\
\hline 43 & 7.5349 & 1.08524 & 2.40543 & 1128.14 & 900.59 \\
\hline 44 & 7.3636 & 1.54108 & 0.11851 & 387.01 & 971.90 \\
\hline 45 & 7.2000 & 0.29265 & -3.37076 & 1854.52 & 1357.85 \\
\hline 46 & 7.0435 & 3.03355 & -0.12055 & 1493.15 & 1387.96 \\
\hline 47 & 6.8936 & 2.11664 & 1.34556 & 1019.09 & 986.18 \\
\hline 48 & 6.7500 & 0.44153 & -1.53776 & 414.67 & 543.56 \\
\hline 49 & 6.6122 & 0.89054 & 0.29160 & 142.25 & 377.70 \\
\hline 50 & 6.4800 & 1.79396 & 1.06231 & 704.18 & 422.46 \\
\hline 51 & 6.3529 & 1.14986 & -0.27166 & 226.15 & 325.34 \\
\hline 52 & 6.2308 & 0.87909 & 0.01307 & 125.22 & 283.13 \\
\hline 53 & 6.1132 & 1.60409 & -0.87897 & 542.00 & 414.36 \\
\hline 54 & 6.0000 & 0.20788 & 1.69652 & 473.26 & 493.06 \\
\hline 55 & 5.8909 & 1.77681 & 0.55324 & 561.03 & 537.48 \\
\hline 56 & 5.7857 & 1.36426 & 0.33134 & 319.30 & 834.52 \\
\hline 57 & 5.6842 & 2.39626 & -2.73815 & 2144.80 & 1218.52 \\
\hline 58 & 5.5862 & 1.92971 & -0.46082 & 637.65 & 889.23 \\
\hline
\end{tabular}


Yousuf Zai et al. / Int. J. of Sustainable Water \& Environmental Systems, 7 (2015) 47-53

\begin{tabular}{|c|c|c|c|c|c|}
\hline 59 & 5.4915 & $\overline{-} .09701$ & 0.75792 & 288.02 & 411.40 \\
\hline 60 & 5.4000 & 0.83639 & 0.67381 & 186.88 & 253.93 \\
\hline 61 & 5.3115 & $\overline{-}-41686$ & -1.05551 & 208.64 & 370.39 \\
\hline 62 & 5.2258 & 1.50926 & 1.60055 & 784.02 & 652.81 \\
\hline 63 & 5.1429 & 2.09387 & -1.14202 & 921.54 & 768.66 \\
\hline 64 & 5.0625 & 1.32889 & 1.52498 & 662.83 & 570.32 \\
\hline 65 & 4.9846 & 0.15776 & 0.38736 & 28.34 & 330.60 \\
\hline 66 & 4.9091 & 1.67990 & -0.43680 & 488.09 & 303.21 \\
\hline 67 & 4.8358 & 0.68540 & -0.91743 & 212.45 & 251.26 \\
\hline 68 & 4.7647 & 0.67047 & -0.41454 & 100.66 & 212.42 \\
\hline 69 & 4.6957 & $\overline{1}-\overline{1.50430}$ & -0.25677 & 377.27 & 254.86 \\
\hline 70 & 4.6286 & 0.11216 & -1.16185 & 220.72 & 217.30 \\
\hline 71 & 4.5634 & $\overline{0}-23544$ & -0.42597 & 38.37 & 185.62 \\
\hline 72 & 4.5000 & 1.42926 & -0.73723 & 418.98 & 219.79 \\
\hline 73 & 4.4384 & 0.33959 & 0.15153 & 22.40 & 202.70 \\
\hline 74 & 4.3784 & 0.37703 & 1.27372 & 285.85 & 335.17 \\
\hline 75 & 4.3200 & 1.27718 & -1.43890 & 599.66 & 651.04 \\
\hline 76 & 4.2632 & 2.49094 & -1.07842 & 1193.58 & 895.52 \\
\hline 77 & 4.2078 & 0.75470 & 1.97602 & 724.82 & 859.87 \\
\hline 78 & 4.1538 & 1.99525 & 1.32324 & 928.58 & 656.43 \\
\hline 79 & 4.1013 & $\overline{-}-44342$ & -0.60864 & 91.86 & 331.28 \\
\hline 80 & 4.0500 & 0.52381 & -0.37004 & 66.63 & 255.52 \\
\hline 81 & 4.0000 & 1.49183 & -1.41558 & 685.17 & 366.09 \\
\hline 82 & 3.9512 & 0.46028 & 0.83785 & 148.04 & 298.64 \\
\hline 83 & 3.9036 & 0.09294 & 0.94133 & 144.95 & 335.43 \\
\hline 84 & 3.8571 & 0.62168 & -2.19273 & 841.52 & 476.10 \\
\hline 85 & 3.8118 & 1.00001 & -0.57414 & 215.40 & 369.50 \\
\hline 86 & 3.7674 & 0.39501 & 1.12786 & 231.35 & 260.48 \\
\hline 87 & 3.7241 & 1.26653 & 0.20460 & 266.65 & 259.32 \\
\hline 88 & 3.6818 & 1.31426 & -0.40274 & 306.09 & 241.01 \\
\hline 89 & 3.6404 & 0.53077 & 0.49102 & 84.70 & 210.97 \\
\hline 90 & 3.6000 & - & 1.35912 & 319.30 & 261.79 \\
\hline
\end{tabular}

\begin{tabular}{|c|c|c|c|c|c|}
\hline & & 0.35182 & & & \\
\hline 91 & 3.5604 & $\overline{1}-\overline{1.31670}$ & 0.70017 & 360.28 & 266.26 \\
\hline 92 & 3.5217 & $\overline{0} .05268$ & 0.42130 & 29.20 & 255.97 \\
\hline 93 & 3.4839 & $\overline{0} .17642$ & -1.77390 & 514.81 & 393.71 \\
\hline 94 & 3.4468 & 1.34941 & 1.31724 & 576.08 & 431.22 \\
\hline 95 & 3.4105 & $\begin{array}{l}- \\
0.36982\end{array}$ & -0.86255 & 142.68 & 322.62 \\
\hline 96 & 3.3750 & $\overline{1}-\mathbf{1 . 0 5 6 1 9}$ & -1.17893 & 405.88 & 262.39 \\
\hline 97 & 3.3402 & 0.66547 & -0.46546 & 106.84 & 154.64 \\
\hline 98 & 3.3061 & $\overline{-} .27686$ & -0.06442 & 13.09 & 59.68 \\
\hline 99 & 3.2727 & 0.37854 & -0.05211 & 23.65 & 77.52 \\
\hline 100 & 3.2400 & 1.15179 & -0.19148 & 220.85 & 163.47 \\
\hline 101 & 3.2079 & $\overline{0} .90088$ & 0.59511 & 188.85 & 228.49 \\
\hline 102 & 3.1765 & 0.66940 & -1.35282 & 369.07 & 225.90 \\
\hline 103 & 3.1456 & 0.33068 & -0.29126 & 31.46 & 119.46 \\
\hline 104 & 3.1154 & $\overline{-}-01686$ & 0.15560 & 3.97 & 84.77 \\
\hline 105 & 3.0857 & $\overline{0} .60566$ & -1.06968 & 244.79 & 142.21 \\
\hline 106 & 3.0566 & 0.59595 & -0.44862 & 90.14 & 176.60 \\
\hline 107 & 3.0280 & 1.06362 & -0.66758 & 255.46 & 253.46 \\
\hline 108 & 3.0000 & $\overline{1.01434}$ & 1.29254 & 437.33 & 289.17 \\
\hline 109 & 2.9725 & $\overline{0} \mathbf{0} 76991$ & -0.11743 & 98.26 & 224.26 \\
\hline 110 & 2.9455 & $\overline{-}-20735$ & -0.94785 & 152.51 & 313.74 \\
\hline 111 & 2.9189 & 2.14389 & -0.65477 & 814.05 & 472.81 \\
\hline 112 & 2.8929 & $\overline{1}-10705$ & 0.72151 & 282.87 & 345.46 \\
\hline 113 & 2.8673 & $\overline{-}-26322$ & -0.30180 & 25.98 & 189.93 \\
\hline 114 & 2.8421 & 0.24581 & -1.37082 & 314.21 & 201.81 \\
\hline 115 & 2.8174 & $\overline{-}-67856$ & 0.67784 & 149.02 & 209.57 \\
\hline 116 & 2.7931 & 1.02301 & -0.74311 & 259.00 & 189.68 \\
\hline 117 & 2.7692 & $\overline{0}-72290$ & 0.39570 & 110.02 & 128.38 \\
\hline 118 & 2.7458 & 0.22591 & -0.12335 & 10.73 & 103.77 \\
\hline 119 & 2.7227 & 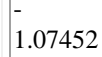 & -0.62128 & 249.57 & 154.77 \\
\hline
\end{tabular}


Yousuf Zai et al. / Int. J. of Sustainable Water \& Environmental Systems, 7 (2015) 47-53

\begin{tabular}{|c|c|c|c|c|c|}
\hline 120 & 2.7000 & 0.07561 & -0.72113 & 85.17 & 218.78 \\
\hline 121 & 2.6777 & 1.33779 & -1.01403 & 456.50 & 303.24 \\
\hline 122 & 2.6557 & 0.10083 & 1.32108 & 284.38 & 254.24 \\
\hline 123 & 2.6341 & $\overline{0}-41618$ & -0.27642 & 40.44 & 138.49 \\
\hline 124 & 2.6129 & $\overline{0}-83233$ & -0.27596 & 124.57 & 117.25 \\
\hline 125 & 2.5920 & 0.88883 & 0.41199 & 155.48 & 130.13 \\
\hline 126 & 2.5714 & 0.75408 & -0.40724 & 118.99 & 102.15 \\
\hline 127 & 2.5512 & $\overline{0}-30385$ & 0.06902 & 15.73 & 67.94 \\
\hline 128 & 2.5313 & 0.41211 & -0.63411 & 92.65 & 82.98 \\
\hline 129 & 2.5116 & 0.45761 & 0.73629 & 121.75 & 111.95 \\
\hline 130 & 2.4923 & 0.50825 & 0.68761 & 118.44 & 129.52 \\
\hline 131 & 2.4733 & $\overline{0} .94741$ & -0.40659 & 172.19 & 132.53 \\
\hline 132 & 2.4545 & 0.62585 & 0.19228 & 69.44 & 126.59 \\
\hline 133 & 2.4361 & $\overline{0}-53962$ & -0.86540 & 168.50 & 164.28 \\
\hline 134 & 2.4179 & 0.92457 & 0.86017 & 258.34 & 198.01 \\
\hline 135 & 2.4000 & 0.79327 & 0.20517 & 108.76 & 215.40 \\
\hline 136 & 2.3824 & 1.38513 & 0.62410 & 373.91 & 260.41 \\
\hline 137 & 2.3650 & 0.23435 & -1.18245 & 235.40 & 208.35 \\
\hline 138 & 2.3478 & 0.42819 & -0.19990 & 36.18 & 97.01 \\
\hline 139 & 2.3309 & 0.18366 & -0.23466 & 14.38 & 80.34 \\
\hline 140 & 2.3143 & $\overline{-}$ & 0.15983 & 204.22 & 147.22 \\
\hline 141 & 2.2979 & $\overline{0} 02814$ & -1.14225 & 211.49 & 150.46 \\
\hline 142 & 2.2817 & 0.21824 & -0.07225 & 8.56 & 91.95 \\
\hline 143 & 2.2657 & 0.29782 & 0.80233 & 118.65 & 73.56 \\
\hline 144 & 2.2500 & 0.38185 & 0.26241 & 34.78 & 68.15 \\
\hline 145 & 2.2345 & $\overline{0}-66160$ & -0.10187 & 72.59 & 88.08 \\
\hline 146 & 2.2192 & 0.72917 & -0.73635 & 173.97 & 141.77 \\
\hline 147 & 2.2041 & 0.42738 & -0.09683 & 31.11 & 341.25 \\
\hline 148 & 2.1892 & 0.55040 & 2.49823 & 1060.15 & 672.33 \\
\hline 149 & 2.1745 & 2.00352 & -0.83532 & 763.32 & 608.24 \\
\hline 150 & 2.1600 & 0.47837 & -0.02525 & 37.18 & 254.71 \\
\hline 151 & 2.1457 & 0.23807 & -0.50854 & 51.08 & 95.29 \\
\hline 152 & 2.1316 & 0.48512 & 0.66519 & 109.81 & 130.78 \\
\hline
\end{tabular}

\begin{tabular}{|c|c|c|c|c|c|}
\hline 153 & 2.1176 & 0.50899 & -1.19724 & 274.18 & 169.45 \\
\hline 154 & 2.1039 & $\overline{0} .31978$ & 0.49713 & 56.60 & 132.62 \\
\hline 155 & 2.0903 & 0.70441 & 0.62199 & 143.06 & 112.01 \\
\hline 156 & 2.0769 & $\overline{0} .66649$ & 0.21987 & 79.79 & 112.78 \\
\hline 157 & 2.0637 & $\overline{0} .09585$ & 0.96764 & 153.17 & 125.27 \\
\hline 158 & 2.0506 & 0.04562 & -0.80095 & 104.26 & 140.89 \\
\hline 159 & 2.0377 & 1.07947 & 0.33955 & 207.45 & 162.12 \\
\hline
\end{tabular}

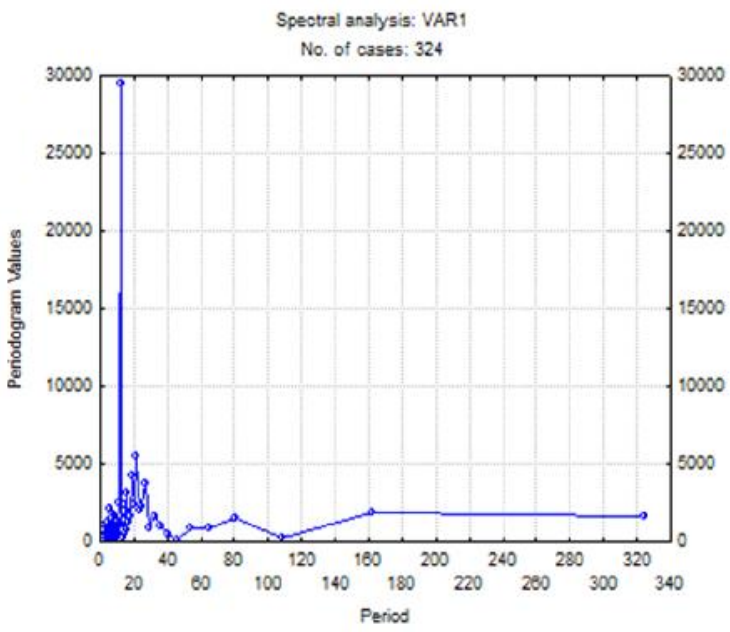

Figure 2. Time Domain Aspect of Periodogram

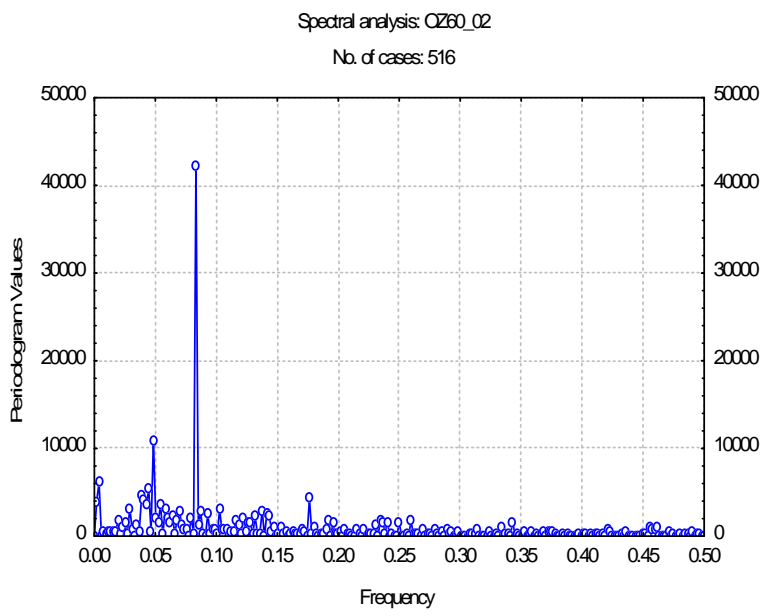

Figure 3. Frequency domain Aspect of Periodogranm

It has been noticed that we have power in each Fourier component, called power spectral density or more simply a power spectrum. Since the power is proportional to the square of the amplitude of an oscillation and since there are both sine and cosine terms $90^{\circ}$ out of phase, the 
power $\mathrm{S}(f)$ at frequency $\mathrm{m} f_{0}$ is
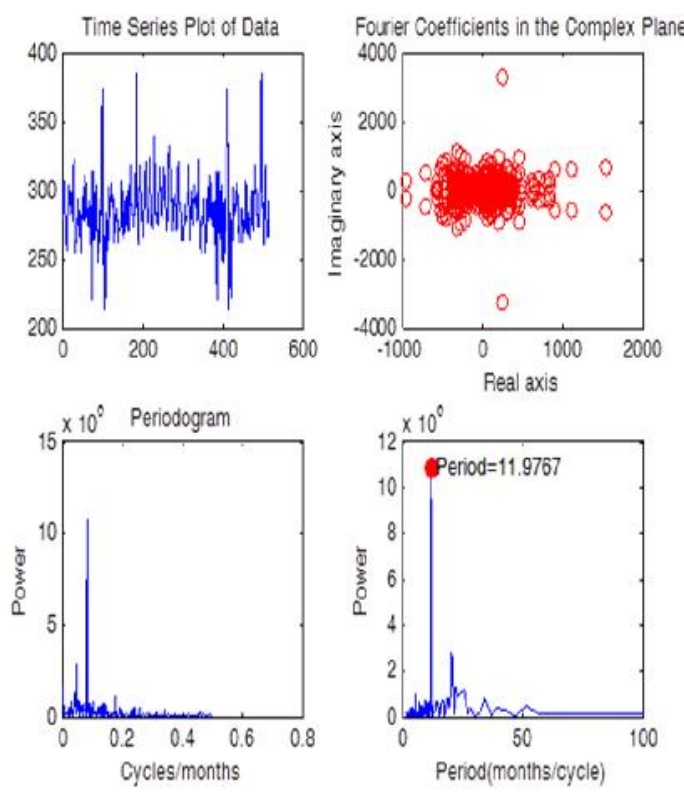

Figure 4. Plot Shows The Temporal Fluctuations, Power Vs Cycles /Months, Fourier Components In Complex Planes And Power Vs Period (Months / Cycle)

$$
\mathrm{S}_{\mathrm{m}}=a^{2}{ }_{m}+b^{2}{ }_{m}
$$

Only relative values of $S_{m}$ are taken. Albeit it could be normalized by various procedures.

Fourier analysis is a mathematical technique used to obtain the frequency spectrum of an a-periodic signals, if the time domain representation is given. Similarly, Fourier series is a technique to decompose the entire series into components and Fourier transform is a technique to reduce the series into components. The periodicity in the ozone layer sequence is found using FFT that is equal to 11.98 as shown in the power spectrum of the ozone data set figure.4. Discrete time Signal processing techniques to collect, store and analyze the data in which Fourier transform, short time Fourier Transform (STFT) or frequencytime spectrograms are found as shown in the above figures.

\section{Wavelet Analysis}

The word wavelet was adopted to express the idea of small waves in an intuitive which is associated with localized waves like function whose values fluctuate in short period, in order to a function be called a wavelet. Wavelet analysis is a particular time-or space-scale representation of signals that has been found in atmospheric application like here in this case for the characterization of ozone concentration.
Wavelet is the name given to a set of mathematical functions used for image Compression, the actual wavelet analysis is a mathematical technique, which is very useful for numerical analysis and manipulation of multidimensional discrete signal sets. Originally applied in geophysics to the analysis of seismic signals, the wavelet transforms were better and broadly formalized thanks to mathematicians, physicists, and engineers efforts. In atmospheric applications, the main characteristic of wavelet technique is the introduction of the time frequency decomposition. When wavelet analysis is used to study a given signal, it is essential to choose the best wavelet representation for the signal under study. In statistical applications, the wavelet transform is linear and covariant under translation and dilatation transform. The wavelet transform can be used in the analysis of non-stationary signals to obtain information on the frequency or scale variations of those signals and to detect its structures localization in time and or in space.

Wavelet analysis is not replacing frequency analysis but it is rather an important refinement and expansion of it. The wave let can also be of great help in uncovering the presence and absence of certain frequencies in a physical system as OLD. Fourier analysis analyses a signal globally, whereas wavelet analysis looks into the signal locally.

\section{Comparison between Fourier Transform and Wavelet}

It is a fact that most of the signals including the ozone layer thickness cover a wide range of frequencies. Instead of transient components thee is a frequently a direct correlation between the characteristic frequency of a given segment of the ozone signal and time duration of that signal. Wavelet analysis has a number of advantages over Fourier analysis they are particularly attractive. Unlike the Fourier transform that generates record averaged values of amplitude and phase for each frequency component or harmonic, $\omega$, the wavelet transform yields a localized, "instantaneous" estimate for the amplitude ans phase of each spectral component in the data set like ozone. This gives wavelet analysis an advantage in the analysis of signals like ozone concentration data set in which amplitudes and phases of the harmonic components are changing rapidly in time and space.

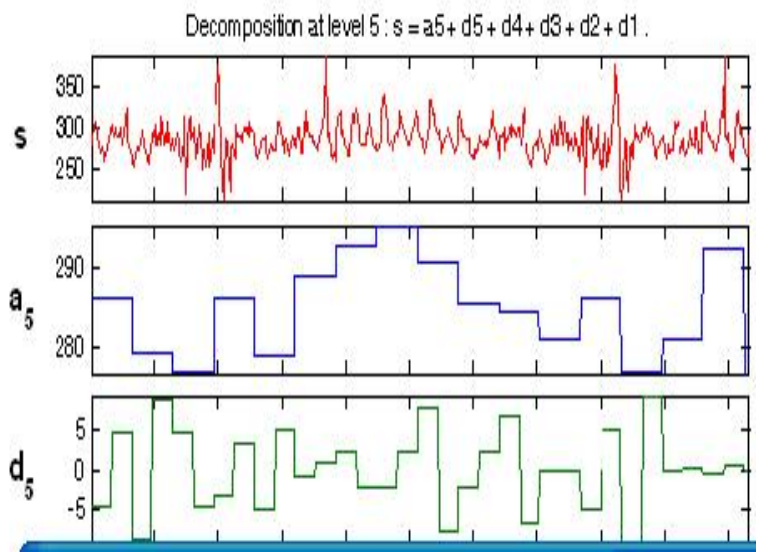

Figure 5. Haar Wavelet with Level 5, Full Decomposition at Level 5. 


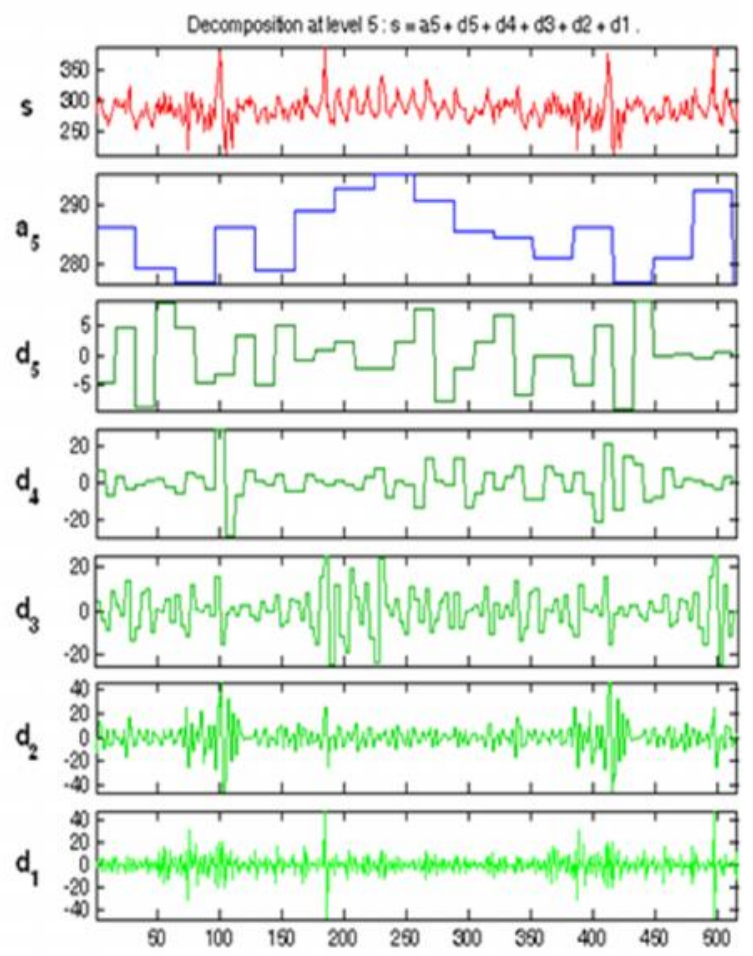

Figure 6. An Ozone Layer Signal Analyzed By Haar Wavelet. A Decomposition of Order 5. The Signal S Lives In $\mathrm{V}_{0}$ And It Is Decomposed Into Its Approximation $A_{5}$ As A Member Of $V_{-5}$ And Increasingly Finer Details $D_{\mathrm{j}}$ Is A Member Of $\mathrm{W}_{-\mathrm{J}}, \mathrm{J}=\mathbf{5 , 4 , 3 , 2 , 1}$.

Indeed the wavelets provide a framework for signal decomposition in the form of a sequence of signals known as approximation signals with decreasing resolution supplemented by a sequence of additional touches called details as illustrated in the ozone signal in figure 5 figure 6.

\section{Conclusion}

In this communication we have compared Fourier analysis and wavelet analysis and found that Wavelet analysis has a number of advantages over Fourier analysis they are particularly attractive. Unlike the Fourier transform that generates record averaged values of amplitude and phase for each frequency component or harmonic, $\omega$, the wavelet transform yields a localized, "instantaneous" estimate for the amplitude ans phase of each spectral component in the data set like ozone.Wavelet transformed spectral analysis is used to find significance periodicities of the series. Also wavelet coherence analysis is used to find the different phases of the periodicities of the OLD data sets

\section{Acknowledgement}

I would like to thank the organizers to provide me the opportunity to present this piece of information before this eminent gathering and local hospitality rendered by them towards me. Also the thanks are due for the staff of geophysical center who took pains in recording the data sets of ozone layer depletion.

\section{References}

[1] Smith C (2001), "Environmental Physics", Routledge , Talor and Francis group, London.

[2] Balter M (1995), "Chernobyl's thyroid cancer toll", Science, 270,1758

[3] Boeker, E., Grondelle R., (1999), "Environmental Physics," 2nd edition, JohnWiley, New York

[4] Walker G (200), “The Hole Story,” New Scientist, 25 March, pp 24-28

[5] Lundstedt H, Liszka L and Muscheter R (2006), Long term Solar Activity with wavelet methods, Ann.Geophys, 24,769-778.

[6] Gardner W H, (2005), "Real Analysis with an Introduction to Wavelets and Applications," Academic Press, USA

[7] Kohno R, Michael L B, and Ghavami M (2007), "Ultra Wideband, Signals and Systems in Communication Engineering," Chapter.6, page \# 164-171, Wiley, New York

[8] Vanden Berg J C, (2004), "Wavelets in Physics." Cambridge University Press, UK

[9] Sprott C J, (2004), "Chaos and Time Series Analysis."Oxford University Press, New York

[10] Addison P. (2002), "The Illustrated Wavelet Transform Handbook, Introductory, Theory and Applications in Science, Engineering, Medicine and Finance" Institute of Physics Publishing, Bristol, UK

[11] Kumar P. and Foufoula-Georgiou. E, (1997), Wavelet Analysis for Geophysical Applications, Rev.Geophys., 35(4), pp.385-412

[12] Mallat, S.(1998), "Wavelet tour of signal processing," Academic Press,

[13] 13. Polygiannakis J, Preka-Papadema P., and Moussas X., (2003), on signal -noise decomposition of time series using the continuous wavelet transform, application to sunspot index, Mon.Not. R., Astron Soc., 343, pp. 725-734.

[14] Torrence C., Compo G P., (1998), A practical guide to wavelet analysis, Bull. Am.Meteorol.Soc.,79, 61-78

[15] Wernik A W.,and Grzesiak M., (1997), Analysis of Ionospheric plasma turbulence with wavelet transform, in Proc. Int. Symp.Plasma 97, editedby Sadoski M. and Rothkaehl. Jarnoltowek, Space Center, Polish Academy of Sciences, Vol.1, p.391

[16] Misiti M and Misiti Y, edr.(2007), "Wavelets and their applications...'ISTE 